\title{
THE SERIAL TEST FOR A NONLINEAR PSEUDORANDOM NUMBER GENERATOR
}

\author{
TAKASHI KATO, LI-MING WU, AND NIRO YANAGIHARA
}

\begin{abstract}
Let $M=2^{w}$, and $G_{M}=\{1,3, \ldots, M-1\}$. A sequence $\left\{y_{n}\right\}, y_{n} \in$ $G_{M}$, is obtained by the formula $y_{n+1} \equiv a \bar{y}_{n}+b+c y_{n} \bmod M$. The sequence $\left\{x_{n}\right\}, x_{n}=y_{n} / M$, is a sequence of pseudorandom numbers of the maximal period length $M / 2$ if and only if $a+c \equiv 1(\bmod 4), b \equiv 2(\bmod 4)$. In this note, the uniformity is investigated by the 2-dimensional serial test for the sequence. We follow closely the method of papers by Eichenauer-Herrmann and Niederreiter.
\end{abstract}

\section{INTRODUCTION}

For generating uniform pseudorandom numbers (denoted as PRN) in the interval $I=[0,1)$, the linear congruential methods are commonly used. Recently several nonlinear methods, especially the inversive congruential one, were proposed and investigated. For a modulus $M$, let

$$
Z_{M}=\{0,1, \ldots, M-1\}=Z / M
$$

In the linear method, a sequence $\left\{y_{n}\right\}$ in $Z_{M}$ is generated by

$$
y_{n+1} \equiv c y_{n}+b(\bmod M), \quad n=0,1, \ldots,
$$

where $c, b \in Z_{M}$. The PRN are obtained by the normalization

$$
x_{n}=y_{n} / M \text {. }
$$

In the inversive method with power of two modulus, let $M=2^{w}$ and

$$
G_{M}=\{1,3, \ldots, M-1\}=\{\text { positive odd integers less than } M\} .
$$

For any $u \in G_{M}$, there is a unique $\bar{u} \in G_{M}$ such that $\bar{u} u \equiv 1 \bmod M$. Now a sequence $\left\{y_{n}\right\}$ in $G_{M}$ is generated by the inversive recursion formula

$$
y_{n+1} \equiv a \bar{y}_{n}+b(\bmod M), \quad n=0,1, \ldots,
$$

in which $a, b \in Z_{M}$ are chosen so that $y_{n} \in G_{M}$ implies $y_{n+1} \in G_{M}$.

In a previous note we have proposed another nonlinear method which is given by the following formula, with the modulus $M=2^{w}$,

$$
y_{n+1} \equiv a \bar{y}_{n}+b+c y_{n}(\bmod M), \quad n=0,1, \ldots,
$$

in which $a, b, c \in Z_{M}$ should be such that $y_{n} \in G_{M}$ implies $y_{n+1} \in G_{M}$. The PRN $\left\{x_{n}\right\}$ is defined by (1.2). In [7], we proved the following Theorem A, which shows that the modified inversive method (1.4) bears close resemblance to (1.3):

Received by the editor October 25, 1994

1991 Mathematics Subject Classification. Primary 65C10; Secondary 11K45.

Key words and phrases. Pseudorandom number generator, the inversive congruential method, power of two modulus, discrepancy, $k$-dimensional serial test, Kloostermann sum. 
Theorem A. Let $M=2^{w}, w \geqq 3$. Then the PRN $\left\{x_{n}\right\}$ derived from (1.4) is purely periodic with period $M / 2$ if and only if

$$
a+c \equiv 1 \quad(\bmod 4) \quad \text { and } b \equiv 2(\bmod 4) .
$$

Now we will study the behavior of these PRN under the 2-dimensional serial test. That is, we will estimate the discrepancy of the PRN. For a dimension $k \geqq 2$ and for $N$ arbitrary points $\mathbf{t}_{0}, \mathbf{t}_{1}, \ldots, \mathbf{t}_{N-1} \in[0,1)^{k}$ we define the discrepancy

$$
D_{N}\left(\mathbf{t}_{0}, \mathbf{t}_{1}, \ldots, \mathbf{t}_{N-1}\right)=\sup _{J}\left|F_{N}(J)-V(J)\right|,
$$

where the supremum is extended over all subintervals $J$ of $[0,1)^{k}, F_{N}(J)$ is $N^{-1}$ times the number of terms among $\mathbf{t}_{0}, \mathbf{t}_{1}, \ldots, \mathbf{t}_{N-1}$ falling into $J$, and $V(J)$ denotes the $k$-dimensional volume of $J$. If $\left\{x_{n}\right\}$ is a sequence of PRN in $[0,1)$ with period $p$, then we consider the points

$$
\mathbf{x}_{n}=\left(x_{n}, x_{n+1}, \ldots, x_{n+k-1}\right) \in[0,1)^{k} \text { for } n=0,1, \ldots, p-1,
$$

and write their discrepancy $D_{p}\left(\mathbf{x}_{0}, \mathbf{x}_{1}, \ldots, \mathbf{x}_{p-1}\right)$ as $D_{p}^{(k)}$.

Theorem 1. Let $M=2^{w}(w \geqq 6)$ and $a, b, c \in Z_{M}$. Suppose $a+c \equiv 1(\bmod 4), b \equiv$ $2(\bmod 4)$ and $a \neq 0$. Then, for the PRN $\left\{x_{n}\right\}$ in Theorem $\mathrm{A}$, we have

(I) If $c$ is an even number, hence $a$ is odd, then

$$
D_{M / 2}^{(2)}<2 K M^{-1 / 2}(\log M)^{2}+1.12 M^{-1 / 2} \log M+1.35 M^{-1 / 2}+4 / M,
$$

with $K=2 /\left\{\left(2^{3 / 2}-1\right) B P\left(J^{2}\right\}\right.$.

(II) If $c$ is odd (hence $a$ is even), then writing $a=2^{t} a^{\prime}, a^{\prime}$ odd, we have

$$
D_{M / 2}^{(2)}<2^{t / 2} M^{-1 / 2}\left\{2 K(\log M)^{2}+(1.12) \log M+1.35\right\}+4 / M+2 L / M^{2},
$$

with $K=2 /\left\{\left(2^{3 / 2}-1\right) B P\left(J^{2}\right\}\right.$ and $L=2^{2 t}\left\{2(t-1)(t+2)^{2}+14(t+6)^{2}\right\}$, assuming that $w \geqq t+6$.

Theorem 2. Let $M=2^{w}, w \geqq 6$. Let $0<r \leqq 2$ and $A(r)=\left(4-r^{2}\right) /\left(8-r^{2}\right)$. Suppose $c \in Z_{M}$ is given.

If $c$ is even, there are more than $A(r) M / 8$ values of $a \in Z_{M}$ such that $a+c \equiv$ $1 \bmod 4$, and for any $b \in Z_{M}$ with $b \equiv 2 \bmod 4$, we have

$$
D_{M / 2}^{(k)} \geqq K^{\prime} M^{-1 / 2} \quad \text { with } K^{\prime}=r /(\pi+2) .
$$

If $c$ is odd, there are more than $A(r) M / 32$ values of $a \in Z_{M}$ such that $a+c \equiv$ $1 \bmod 4$, and for any $b \in Z_{M}$ with $b \equiv 2 \bmod 4$, we have

$$
D_{M / 2}^{(k)} \geqq\left(2 K^{\prime} / 3\right) M^{-1 / 2} \quad \text { with } K^{\prime}=r /(\pi+2) .
$$

Our proofs of Theorems 1 and 2 are almost the same as in [9, Theorem 2], [6, Theorems 1-2], respectively. The lattice structure of the sequence generated by (1.4) will be studied in another paper. 
THE SERIAL TEST FOR A NONLINEAR PSEUDORANDOM NUMBER GENERATOR 763

\section{Proof of Theorem 1}

We closely follow the method in [9, p.141]. Let $M=2^{w}, w \geqq 6$.

Suppose $m=2^{f}$, with a positive integer $f$, be given. For $k \geqq 1$, let $C_{k}(m)$ be the set of all nonzero lattice points $\left(h_{1}, \ldots, h_{k}\right) \in Z^{k}$ with $-m / 2<h_{j} \leqq m / 2$, for $1 \leqq j \leqq k$. We put

$$
r(h, m)= \begin{cases}1 & \text { for } h=0, \\ m \sin (\pi|h| / m) & \text { for } h \in C_{1}(m),\end{cases}
$$

and for $\mathbf{h}=\left(h_{1}, \ldots, h_{k}\right) \in C_{k}(m)$ we define

$$
r(\mathbf{h}, m)=\prod_{j=1}^{k} r\left(h_{j}, m\right) .
$$

For real $s$ we write $e(s)=e^{2 \pi i s}$. For $x, y \in \mathbf{R}^{k}, x \cdot y$ denotes the inner product. We put, for integers $u, v$,

$$
S(u, v ; m)=\sum_{n \in G_{m}} e((u n+v \bar{n}) / m),
$$

in which $\bar{n} \in G_{m}$ denotes the number such that $\bar{n} n \equiv 1(\bmod m)$. This sum has the following properties $[12,9]$ :

$$
\begin{gathered}
S(u, v ; m)=S(1, u v ; m) \text { if } u \text { is odd, } \\
S(u, v ; m)=0 \text { if } u+v \equiv 1(\bmod 2), \\
S(u, v ; m)=2^{d} S\left(u / 2^{d}, v / 2^{d} ; 2^{f-d}\right) \text { if } u \equiv v \equiv 0 \bmod 2^{\mathrm{d}} \text { and } d<f,
\end{gathered}
$$

where in $(2.2)$ and $(2.3)$ we assume that $f \geqq 2$. Further (see $[9$, p.140]),

$$
\begin{gathered}
|S(1, v ; 8)|= \begin{cases}4 & \text { if } v \equiv 3 \bmod 4, \\
0 & \text { otherwise }\end{cases} \\
|S(1, v ; 16)|= \begin{cases}4 \sqrt{2} & \text { if } v \equiv 1 \bmod 4, \\
0 & \text { otherwise },\end{cases} \\
|S(1, v ; 32)| \leqq \begin{cases}8 \sqrt{2+\sqrt{2}} & \text { if } v \equiv 5 \bmod 8, \\
0 & \text { otherwise. }\end{cases}
\end{gathered}
$$

For $f \geqq 6$, we have

$$
\left|S\left(1, v ; 2^{f}\right)\right| \leqq \begin{cases}2^{(f+3) / 2} & \text { if } v \equiv 1 \bmod 8 \\ 0 & \text { otherwise. }\end{cases}
$$

The following lemmas are from [9, p.136 and p.140]. 
Lemma 2.1. Let $m \geqq 2$ be an integer and let $\mathbf{y}_{0}, \mathbf{y}_{1}, \ldots, \mathbf{y}_{N-1} \in Z^{k}$ be lattice points all of whose coordinates are in $[0, m)$. Then the discrepancy of the points $\mathbf{t}_{n}=\mathbf{y}_{n} / m, 0 \leqq n \leqq N-1$, satisfies

$$
D_{N}\left(\mathbf{t}_{0}, \mathbf{t}_{1}, \ldots, \mathbf{t}_{N-1}\right) \leqq \frac{k}{m}+\frac{1}{N} \sum_{\mathbf{h} \in C_{k}(m)} \frac{1}{r(\mathbf{h}, m)}\left|\sum_{n=0}^{N-1} e\left(\mathbf{h} \cdot \mathbf{t}_{n}\right)\right|
$$

Lemma 2.2. Let $m=2^{f}$. For $f \geqq 6$ and $r$ odd, we have

$$
\sum_{k \in C_{1}(m), k \equiv r(\bmod 8)} \csc \left(\frac{\pi|k|}{m}\right)<\frac{(f+1)(\log 2)}{4 \pi} m+0.2126 m
$$

and for $f \geqq 3$ we have

$$
\sum_{k \in C_{1}(m), k \text { odd }} \csc \left(\frac{\pi|k|}{m}\right)<\frac{(f+1)(\log 2)}{\pi} m+0.3024 m .
$$

Now we prove Theorem 1. Since $\left\{y_{0}, y_{1}, \ldots, y_{M / 2-1}\right\}=G_{M}$, we have

$$
\left\{\left(y_{n}, y_{n+1}\right) ; 0 \leqq n \leqq M / 2-1\right\}=\left\{(n, a \bar{n}+b+c n) ; n \in G_{M}\right\} .
$$

Lemma 2.1 yields

$$
D_{M / 2}^{(2)} \leqq \frac{2}{M}+\frac{2}{M} \sum_{\mathbf{h} \in C_{2}(M)} \frac{|S(\mathbf{h})|}{r(\mathbf{h}, M)}
$$

where for $\mathbf{h}=\left(h_{1}, h_{2}\right) \in C_{2}(M)$ we have

$$
|S(\mathbf{h})|=\left|\sum_{n \in G_{M}} e\left(\frac{\left(h_{1}+h_{2} c\right) n+h_{2} a \bar{n}+h_{2} b}{M}\right)\right|=\left|S\left(h_{1}+h_{2} c, h_{2} a ; M\right)\right| .
$$

Now $\operatorname{gcd}\left(h_{1}, h_{2}, M\right)=2^{d}$ with $0 \leqq d \leqq w-1$, so splitting up the following sum according to the value of $d$, we get

$$
\sum:=\sum_{\mathbf{h} \in C_{2}(M)} \frac{|S(\mathbf{h})|}{r(\mathbf{h}, M)}=\sum_{d=0}^{w-1} T_{d}
$$

with

$$
T_{d}=\sum_{\mathbf{h}} \frac{\left|S\left(h_{1}+h_{2} c, h_{2} a ; M\right)\right|}{r(\mathbf{h}, M)},
$$

where the last sum is extended over all $\mathbf{h}=\left(h_{1}, h_{2}\right) \in C_{2}(M)$ with $\operatorname{gcd}\left(h_{1}, h_{2}, M\right)=$ $2^{d}$. It follows immediately that

$$
T_{w-1}=1+\frac{1}{2 M}
$$


THE SERIAL TEST FOR A NONLINEAR PSEUDORANDOM NUMBER GENERATOR 765

Now consider $0 \leqq d \leqq w-2$. Write $k_{1}=h_{1} / 2^{d}, k_{2}=h_{2} / 2^{d}$. If one of $k_{1}$ or $k_{2}$ is even, then (2.3) and (2.2) imply $S\left(h_{1}+h_{2} c, h_{2} a ; M\right)=0$. Thus it suffices to suppose that both $k_{1}$ and $k_{2}$ are odd.

We divide the proof into two cases (I) and (II):

(I) c is an even number, hence $a$ is odd. In this case, (2.3) and (2.1) yield

$$
S\left(h_{1}+h_{2} c, h_{2} a ; M\right)=2^{d} S\left(1,\left(k_{1}+k_{2} c\right) k_{2} a ; 2^{w-d}\right) .
$$

Thus we obtain

$$
T_{d}=2^{d} \sum_{\substack{k_{1}, k_{2} \in C_{1}\left(2^{w-d}\right) \\ k_{1}, k_{2} \text { odd }}} \frac{\left|S\left(1,\left(k_{1}+k_{2} c\right) k_{2} a ; 2^{w-d}\right)\right|}{r\left(k_{1} 2^{d}, M\right) r\left(k_{2} 2^{d}, M\right)} .
$$

For $0 \leqq d \leqq w-6$, we use $(2.7)$ to get

$$
T_{d} \leqq 2^{(w+d+3) / 2} \sum\left\{r\left(k_{1} 2^{d}, M\right) r\left(k_{2} 2^{d}, M\right)\right\}^{-1}
$$

with the sum over odd numbers $k_{1}, k_{2} \in C_{1}\left(2^{w-d}\right)$ such that $\left(k_{1}+k_{2} c\right) k_{2} a \equiv 1$ $(\bmod 8)$, that is, $k_{1}+k_{2} c \equiv k_{2} a(\bmod 8)$, i.e.,

$$
k_{1} \equiv k_{2}(a-c) \quad(\bmod 8) .
$$

Thus we have

$$
T_{d} \leqq 2^{(-3 w+d+3) / 2} \sum_{\substack{k_{2} \in C_{1}\left(2^{w-d}\right) \\ k_{2} \text { odd }}} \csc \left(\frac{\pi\left|k_{2}\right|}{2^{w-d}}\right) \sum_{\substack{k_{1} \in C_{1}\left(2^{w-d}\right) \\ k_{1} \equiv k_{2}(a-c)(\bmod 8)}} \csc \left(\frac{\pi\left|k_{1}\right|}{2^{w-d}}\right) .
$$

Together with (2.8) and (2.9), this yields

$$
\begin{aligned}
T_{d} & \leqq 2^{(w-3 d+3) / 2}\left\{\frac{(w-d+1) \log 2}{4 \pi}+0.2126\right\}\left\{\frac{(w-d+1) \log 2}{\pi}+0.3024\right\} \\
& <2^{(w-3 d+3) / 2}\left\{\frac{(\log M)^{2}}{4 \pi^{2}}+0.127 \log M+0.1401+0.0122 d^{2}\right\}
\end{aligned}
$$

Therefore, as in [9, p.142],

$$
\sum_{d=0}^{w-6} T_{d}<M^{1 / 2}\left\{K(\log M)^{2}+0.56 \log M+0.675\right\}-\frac{876}{M},
$$

with $K=2 /\left\{\left(2^{3 / 2}-1\right) \pi^{2}\right\}$.

For $d=w-5$, we get from (2.6) and (2.13)

$$
T_{w-5} \leqq 2^{-w-2} \sqrt{2+\sqrt{2}} \sum_{\substack{k_{2} \in C_{1}(32) \\ k_{2} \text { odd }}} \csc \left(\frac{\pi\left|k_{2}\right|}{32}\right) \sum_{\substack{k_{1} \in C_{1}(32) \\ k_{1} \equiv 5 k_{2}(a-c)(\bmod 8)}} \csc \left(\frac{\pi\left|k_{1}\right|}{32}\right),
$$


in which we note that, in the second sum, $k_{1} \equiv k_{2}(5 a-c) \equiv 5 k_{2}(a-c) \bmod 8$, since $\mathrm{c}$ is even. As in [9, p.142], by distinguishing the cases $a-c \equiv 1$ or $a-c \equiv 5$ $\bmod 8$, we have

$$
T_{w-5}<240 / M
$$

Similarly, using (2.4), (2.5) and (2.13), we get

$$
T_{w-4}<60 / M, \quad T_{w-3}<14 / M
$$

Since $|S(1, v ; 4)|=2$ for $v$ odd, it follows from (2.12) that

$$
T_{w-2}=4 / M
$$

By combining $(2.11)$ and $(2.16,17,18,19)$, we get

$$
\sum:=\sum_{d=0}^{w-1} T_{d}<M^{1 / 2}\left\{K(\log M)^{2}+0.56 \log M+0.675\right\}+1
$$

with the constant $\mathrm{K}$ in (2.16). The desired result follows from (2.10).

(II) $c$ is an odd number, hence $a(\neq 0)$ is even, $a \in Z_{M}$. Put $a=2^{t} a^{\prime}, a^{\prime}$ odd. Consider some $T_{d}, 0 \leqq d \leqq w-2$.

We always assume that both $k_{j}=h_{j} / 2^{d}, j=1,2$, are odd. Put $2^{s}=$ $\operatorname{gcd}\left(k_{1}+k_{2} c, a, 2^{w-d-1}\right)$, and $r_{1}=\left(k_{1}+k_{2} c\right) / 2^{s}, r_{2}=k_{2} a / 2^{s}$.

(II-1) Suppose $t \geqq w-d-1$. If $s<w-d-1$, then

$$
S(\mathbf{h})=S\left(h_{1}+h_{2} c, h_{2} a ; M\right)=2^{d+s} S\left(r_{1}, r_{2} ; 2^{w-d-s}\right)=0
$$

by (2.2), since $r_{1}$ is odd and $r_{2}$ is even. If $s=w-d-1$, then

$$
S(\mathbf{h})=2^{d} 2^{w-d-1} S\left(r_{1}, r_{2} ; 2\right)=2^{w-1}=M / 2 .
$$

If $w-d \geqq 3$, then

$$
\begin{aligned}
T_{d} & =\frac{M}{2} \sum_{\substack{k_{1}+k_{2} \equiv 0 \text { mod } 2^{w-d-1} \\
k_{1}, k_{2} \text { odd }}} \frac{1}{r\left(k_{1} 2^{d}, M\right) r\left(k_{2} 2^{d}, M\right)} \\
& =\frac{1}{2 M} \sum_{\substack{k_{2} \in C_{1}\left(2^{w-d}\right) \\
k_{2} \text { odd }}} \csc \left(\frac{\pi\left|k_{2}\right|}{2^{w-d}}\right) \sum_{\substack{k_{1} \in C_{1}\left(2^{w-d}\right) \\
k_{1} \equiv-k_{2} c \bmod 2^{w-d-1}}} \csc \left(\frac{\pi\left|k_{1}\right|}{2^{w-d}}\right) \\
& \leqq \frac{1}{2 M}\left\{\frac{(w-d+1) \log 2}{\pi}+0.3024\right\}^{2} 2^{2(w-d)}
\end{aligned}
$$

by Lemma 2.2 . Since $3 \leqq w-d \leqq t+1$, we have

$$
T_{d} \leqq \frac{2^{2 t+1}}{M}\left\{\frac{(t+2) \log 2}{\pi}+0.3024\right\}^{2} .
$$


THE SERIAL TEST FOR A NONLINEAR PSEUDORANDOM NUMBER GENERATOR 767

If $w-d=2$, then

$$
T_{w-2} \leqq 4 \frac{\csc ^{2}(\pi / 4)}{2 M}=\frac{4}{M}
$$

Hence,

$$
\begin{aligned}
\sum_{w-2 \geqq d \geqq w-t-1} T_{d} & =T_{w-2}+\sum_{w-3 \geqq d \geqq w-t-1} T_{d} \\
& \leqq \frac{4}{M}+\frac{(t-1) 2^{2 t+1}}{M}\left\{\frac{(t+2) \log 2}{\pi}+0.3024\right\}^{2},
\end{aligned}
$$

in which the second term does not appear if $t=1$.

(II-2) Now suppose $1 \leqq t \leqq w-d-2$.

We define $s$ and $r_{1}, r_{2}$ as above. Obviously, $s \leqq t$, hence $w-d-1-s \geqq 1$. Thus one of $r_{1}$ or $r_{2}$ must be odd. If one of $r_{1}$ or $r_{2}$ is even,

$$
S(\mathbf{h})=S\left(h_{1}+h_{2} c, h_{2} a ; M\right)=2^{d+s} S\left(r_{1}, r_{2} ; 2^{w-d-s}\right)=0 .
$$

Hence both $r_{1}$ and $r_{2}$ must be odd, which implies $s=t$.

Let $d \leqq w-t-6$. We argue as in the case $d \leqq w-6$ of (I), with $w-t$ instead of $w$; we obtain

$$
\begin{aligned}
& T_{d} \leqq 2^{(-3 w+d+t+3) / 2} \sum_{\substack{k_{2} \in C_{1}\left(2^{w-d}\right) \\
k_{2} \text { odd }}} \csc \left(\frac{\pi\left|k_{2}\right|}{2^{w-d}}\right) \sum_{\substack{k_{1} \in C_{1}\left(2^{w-d}\right), k_{1} \text { odd } \\
r_{1} r_{2} \equiv 1(\bmod 8)}} \csc \left(\frac{\pi\left|k_{1}\right|}{2^{w-d}}\right) \\
& =2^{(-3 w+d+t+3) / 2} \sum_{\substack{k_{2} \in C_{1}\left(2^{w-d}\right) \\
k_{2} \text { odd }}} \csc \left(\frac{\pi\left|k_{2}\right|}{2^{w-d}}\right) \sum_{\substack{k_{1} \in C_{1}\left(2^{w-d}\right), k_{1} \text { odd } \\
r_{1} \equiv r_{2}(\bmod 8)}} \csc \left(\frac{\pi\left|k_{1}\right|}{2^{w-d}}\right) \\
& =2^{(-3 w+d+t+3) / 2} \sum_{\substack{k_{2} \in C_{1}\left(2^{w-d}\right) \\
k_{2} \text { odd }}} \csc \left(\frac{\pi\left|k_{2}\right|}{2^{w-d}}\right) \sum_{\substack{k_{1} \in C_{1}\left(2^{w-d}\right), k_{1} \text { odd } \\
k_{1} \equiv k_{2}(a-c)\left(\bmod 8 \cdot 2^{t}\right)}} \csc \left(\frac{\pi\left|k_{1}\right|}{2^{w-d}}\right) \\
& \leqq 2^{(-3 w+d+t+3) / 2} \sum_{\substack{k_{2} \in C_{1}\left(2^{w-d}\right) \\
k_{2} \text { odd }}} \csc \left(\frac{\pi\left|k_{2}\right|}{2^{w-d}}\right) \sum_{\substack{k_{1} \in C_{1}\left(2^{w-d}\right), k_{1} \text { odd } \\
k_{1} \equiv k_{2}(a-c)(\bmod 8)}} \csc \left(\frac{\pi\left|k_{1}\right|}{2^{w-d}}\right) \\
& \leqq 2^{(w-3 d+t+3) / 2}\left\{\frac{(w-d+1) \log 2}{4 \pi}+0.2126\right\}\left\{\frac{(w-d+1) \log 2}{\pi}+0.3024\right\} \\
& \leqq 2^{(w-3 d+t+3) / 2}\left\{\frac{(\log M)^{2}}{4 \pi^{2}}+(0.127) \log M+0.1401+0.0122 d^{2}\right\},
\end{aligned}
$$

since the set $\left\{k_{1} ; k_{1} \equiv k_{2}(a-c)\left(\bmod 8 \cdot 2^{t}\right)\right\}$ is contained in $\left\{k_{1} ; k_{1} \equiv\right.$ $\left.k_{2}(a-c)(\bmod 8)\right\}$. Hence we obtain, as in [9, p.142],

$$
\sum_{d=0}^{w-t-6} T_{d}<2^{t / 2} M^{1 / 2}\left\{K(\log M)^{2}+0.56 \log M+0.675\right\}-876 / M,
$$

with $K=2 /\left\{\left(2^{3 / 2}-1\right) \pi^{2}\right\}$. 
For $d=w-t-5$, we have as in [9, p.142], with $r_{1}$ and $r_{2}$ as above,

$$
\begin{aligned}
& T_{w-t-5} \leqq 2^{-w-2} \sqrt{2+\sqrt{2}} \sum_{\substack{k_{2} \in C_{1}\left(2^{t+5}\right) \\
k_{2} \text { odd }}} \csc \left(\frac{\pi\left|k_{2}\right|}{2^{t+5}}\right) \sum_{\substack{k_{1} \in C_{1}\left(2^{t+5}\right), k_{1} \text { odd } \\
r_{1} r_{2} \equiv 5(\bmod 8)}} \csc \left(\frac{\pi\left|k_{1}\right|}{2^{t+5}}\right) \\
& \leqq 2^{-w-2} \sqrt{2+\sqrt{2}} \sum_{\substack{k_{2} \in C_{1}\left(2^{t+5}\right) \\
k_{2} \text { odd }}} \csc \left(\frac{\pi\left|k_{2}\right|}{2^{t+5}}\right) \sum_{\substack{k_{1} \in C_{1}\left(2^{t+5}\right), k_{1} \text { odd } \\
k_{1} \equiv k_{2}(5 a-c)(\bmod 8)}} \csc \left(\frac{\pi\left|k_{1}\right|}{2^{t+5}}\right)
\end{aligned}
$$

since $\left\{k_{1} ; r_{1} r_{2} \equiv 5(\bmod 8)\right\}=\left\{k_{1} ; k_{1}+k_{2} c \equiv 5 k_{2} a \quad\left(\bmod 8 \cdot 2^{t}\right)\right\}$ is contained in $\left\{k_{1} ; k_{1} \equiv k_{2}(5 a-c)(\bmod 8)\right\}$. Thus we get

$$
T_{w-t-5}<(t+6)^{2} 2^{2 t+3} / M .
$$

Similarly, using (2.4), (2.5), we get

$$
T_{w-t-4}<(t+5)^{2} 2^{2 t} / M, \quad T_{w-t-3}<(t+4)^{2} 2^{2 t} / M .
$$

Since $|S(1, v ; 4)|=2$ for $v$ odd, it follows that

$$
T_{w-t-2} \leqq(t+3)^{2} 2^{2 t+2} / M .
$$

By (2.11), (2.20), (2.21), (2.22), (2.23), (2.24), we obtain

$$
\sum_{d=0}^{w-1} T_{d}<2^{t / 2} M^{1 / 2}\left\{K(\log M)^{2}+0.56 \log M+0.675\right\}+1+L / M,
$$

with $K=2 /\left\{\left(2^{3 / 2}-1\right) \pi^{2}\right\}$ and $L=2^{2 t}\left\{2(t-1)(t+2)^{2}+14(t+6)^{2}\right\}$. Thus, the desired result follows from (2.10).

\section{Proof of Theorem 2}

The proof is almost the same as in [6].

When $c$ is an even number. Calculating as in $[6$, p.778], putting $\mathbf{h}=(1,1,0, \ldots, 0)$, we have

$$
(\pi+2) M D_{M / 2}^{(k)} \geqq\left|\sum e\left(\frac{y_{n}+y_{n+1}}{M}\right)\right|=|S(1+c, a ; M)|=|S(1,(1+c) a ; M)| .
$$

By [6, Lemma 4], there exist more than $A(r) M / 8$ values of $(1+c) a \in Z_{M}$ such that $(1+c) a \equiv 1(\bmod 8)$, and $|S(1,(1+c) a ; M)| \geqq r M^{1 / 2}$. Then $a \equiv 1+c(\bmod 8)$, hence $a+c \equiv 1+2 c \equiv 1(\bmod 4)$.

When $c$ is odd. If $c=1+8 k$, then put $\mathbf{h}=(3,1,0, \ldots, 0)$ and get

$$
\begin{gathered}
3(\pi+2) M D_{M / 2}^{(k)} \geqq\left|\sum e\left(\frac{3 y_{n}+y_{n+1}}{M}\right)\right|=|S(3+c, a ; M)| \\
=4|S(1+2 k, a / 4 ; M / 4)| \geqq 4 r(M / 4)^{1 / 2}=2 r M^{1 / 2}
\end{gathered}
$$


THE SERIAL TEST FOR A NONLINEAR PSEUDORANDOM NUMBER GENERATOR 769

for more than $A(r) M / 32$ values of $(1+2 k) a / 4$ with $(1+2 k) a / 4 \equiv 1$, i.e., $a / 4 \equiv 1+2 k$ $\bmod 8$. Then $a \equiv 4+8 k=3+c$, hence $a+c \equiv-3+2 a \equiv 1 \bmod 4$.

If $c=3+4 k$, then put $\mathbf{h}=(-1,1,0, \ldots, 0)$ and get

$$
\begin{gathered}
(\pi+2) M D_{M / 2}^{(k)} \geqq\left|\sum e\left(\frac{-y_{n}+y_{n+1}}{M}\right)\right|=|S(c-1, a ; M)| \\
=2|S(1+2 k, a / 2 ; M / 2)| \geqq 2 r(M / 2)^{1 / 2}=\sqrt{2} r M^{1 / 2}
\end{gathered}
$$

for more than $A(r) M / 16$ values of $(1+2 k) a / 2$ with $(1+2 k) a / 2 \equiv 1$, i.e., $a / 2 \equiv 1+2 k$ $\bmod 8$. Then $a \equiv 2+4 k=c-1$, hence $a+c \equiv 1+2 a \equiv 1 \bmod 4$.

If $c=5+8 k$, then put $\mathbf{h}=(-1,1,0, \ldots, 0)$ and get

$$
(\pi+2) M D_{M / 2}^{(k)} \geqq|S(c-1, a ; M)|=4|S(1+2 k, a / 4 ; M / 4)| \geqq 2 r M^{1 / 2}
$$

for more than $A(r) M / 32$ values of $(1+2 k) a / 4$ with $(1+2 k) a / 4 \equiv 1$, i.e., $a / 4 \equiv 1+2 k$ $\bmod 8$. Then $a \equiv 4+8 k=c-1$, hence $a+c \equiv 1+2 a \equiv 1 \bmod 4$.

\section{REFERENCES}

1. J. Eichenauer-Herrmann, Inversive congruential pseudorandom numbers avoid the planes, Math. Comp. 56 (1991), 297-301. MR 91k:65021

2. J. Eichenauer-Herrmann, Statistical independence of a new class of inversive congruential pseudorandom numbers, Math. Comp. 60 (1993), 375-384. MR 93d:65011

3. J. Eichenauer-Herrmann, On generalized inversive congruential pseudorandom numbers, Math. Comp. 63 (1994), 293-299. MR 94k:11088

4. J. Eichenauer-Herrmann, H. Grothe, H. Niederreiter, and A. Topuzoglu, On the lattice structure of a nonlinear generator with modulus $2^{\alpha}$, J. Comp. Appl. Math. 31 (1990), 81-85. MR 91j:65012

5. J. Eichenauer, J. Lehn, and A. Topuzoglu, A nonlinear congruential pseudorandom number generator with power of two modulus, Math. Comp. 51 (1988), 757-759. MR 89i:65007

6. J. Eichenauer-Herrmann and H. Niederreiter, Lower bounds for the discrepancy of inversive congruential pseudorandom numbers with power of two modulus, Math. Comp. 58 (1992), 775-779. MR 92i:65018

7. T. Kato, L.-M. Wu, and N. Yanagihara, On a nonlinear congruential pseudorandom number generator, Math. Comp. 65 (1996) (to appear).

8. D. E. Knuth, The Art of Computer Programming Vol.2: Seminumerical Algorithms, 2nd Ed., Addison-Wesley, Reading, Mass., 1981. MR 83i:68003

9. H. Niederreiter, The serial test for congruential pseudorandom numbers generated by inversions, Math. Comp. 52 (1989), 135-144. MR 90e:65008

10. H. Niederreiter, Recent trends in random number and random vector generation, Ann. Oper. Res. 31 (1991), 323-345. MR 92h:65010

11. H. Niederreiter, Random number generation and quasi-Monte Carlo methods, SIAM, Philadelphia, PA, 1992. MR 93h:65008

12. H. Salié, Über die Kloostermanschen Summen S(u,v;q), Math. Z. 34 (1932), 91-109.

Department of Mathematics, Faculty of Education, Chiba University. 1-33 YayoiCHO, ChiBA City, 263 JAPAN

Department of Mathematics, Faculty of Science, Chiba University, 1-33 Yayoi-cho, Chiba City, 263 JaPAN

Department of Mathematics, Faculty of Science, Chiba University, 1-33 Yayoi-cho, ChiBA City, 263 JAPAN

E-mail address: yanagi@math.s.chiba-u.ac.jp 